\title{
FABIENNE ORIOT
}

\section{EVELYNE MISIASZEK}

Université de Toulouse, Toulouse Business School

\section{Le Balanced Scorecard au filtre d'une PME française}

\author{
Ou pourquoi les PME préfèrent \\ le « sur-mesure »
}

Confrontées à la nécessité d'une prise de décision rapide dans un environnement concurrentiel et incertain, de plus en plus de PME ont désormais besoin de mettre en place des systèmes de management de leur performance stratégique. Bien que conçu à l'origine pour les grandes entreprises, le Balanced Scorecard pourrait offrir une réponse à ce besoin. Une étude de cas conduite dans une PME française questionne d'une part, l'applicabilité de ce modèle normatif dans un contexte spécifique de redressement et esquisse d'autre part, le portrait d'un tableau de bord stratégique "sur-mesure » modelé sur les besoins du dirigeant de cette PME. 


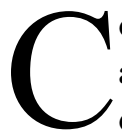
onfrontées à une concurrence vive au niveau mondial et à la nécessité d'une prise de décision rapide dans un environnement incertain et changeant, de plus en plus de PME expriment désormais le besoin, pour survivre et prospérer, de disposer de systèmes de management de leur performance stratégique (St-Pierre et Delisle, 2006). L'étude de ces systèmes répond à un enjeu économique et social majeur dans la mesure où les PME représentent une force vitale de l'économie et de l'emploi dans la plupart des pays industrialisés (Marchand, 2009). Notre contribution s'inscrit dans cette perspective et initie une réflexion exploratoire sur les besoins des PME en matière de systèmes de management de la performance stratégique. Ces systèmes sont définis par Chenhall (2005) comme une combinaison de mesures financières et non financières ayant pour objectif d'aider les dirigeants à maîtriser leurs priorités stratégiques et leurs avantages compétitifs. Le Balanced Scorecard (BSC), formalisé par Kaplan et Norton (1992), est considéré comme le système de management de la performance stratégique le mieux connu des chercheurs et des praticiens (Chenhall, 2005). Ce modèle peut-il constituer une réponse aux besoins des PME ?

Dans une première partie, nous interrogeons l'applicabilité du BSC aux PME à partir d'une analyse synthétique de la littérature. Dans une deuxième partie, nous présentons une étude de cas, conduite auprès d'une PME française. Dans une troisième partie, nous exposons les enseignements de ce cas.

\section{I - LE BSC À L'ÉPREUVE DES PME : EXPLORER L'APPLICABILITÉ DU MODĖLE}

Le BSC a d'abord été présenté dans un article de la Harvard Business Review (Kaplan et Norton, 1992), puis diffusé largement par plusieurs autres articles et ouvrages (Kaplan, 2009) ${ }^{1}$. Conçu à l'origine pour de grandes entreprises du secteur marchand, il convient de se demander s'il est également applicable aux PME.

\section{Le silence discret de Kaplan et Norton}

Kaplan et Norton (2001, p. 369) affirment que le BSC est applicable aux « petites entreprises » mais n'y consacrent aucun développement particulier. Parmi les très nombreux cas d'application dont ils rendent compte, il n'existe, à notre connaissance, qu'un seul cas de succès d'implantation de BSC en petite structure, chez Southern Garden Citrus (Kaplan et Norton, 2001, p. 19), entreprise de 175 salariés. Succinctement décrite, cette expérience de «PME », biaisée par le fait qu'il s'agit d'une filiale d'un grand groupe agroalimentaire, ne donne malheureusement aucune indication sur la démarche de conception à suivre dans ce type de contexte.

Plus récemment, Kaplan (2009) souligne que les organisations des secteurs public et non marchand constituent des opportunités futures d'application mais leur silence perdure sur les PME à proprement parler. Selon nous, ce silence s'explique par le fait que Kaplan et Norton considèrent les PME comme des « grandes entreprises en modèles réduits » (Julien, 2005), auxquelles il serait

1. Kaplan (2009) répertorie les publications majeures de Kaplan et Norton sur le BSC, les principales évolutions du modèle et ses fondements conceptuels. 
donc possible d'appliquer le BSC tel quel. En effet, le BSC est porté par un discours (Méric, 2003), qui le présente comme un modèle universel et générique de performance, échappant à la contingence, parce que suffisamment standard et prêt à l'emploi, pour ne nécessiter qu'un effort d'adaptation minime en fonction des contextes (Bourguignon et al., 2002 ; Choffel et Meyssonier, 2005).

Au-delà de leur diversité, les PME, se différencient pourtant par quelques caractéristiques fondamentales, qui induisent des modes de gestion différents des grandes entreprises (Julien, 2005 ; Torres, 2007), et qui conduisent à s'interroger sur le fait qu'elles aient les mêmes besoins que ces dernières en matière de systèmes de management de la performance. Tout d'abord, le dirigeant, souvent aussi propriétaire, joue un rôle dominant dans le management (Julien, 2005); il est au centre des systèmes de coordination informels avec les acteurs internes et externes (Torres, 2007). Ensuite, les structures sont relativement simples et les ressources limitées (Marchesnay, 1993). Enfin, la survie des PME est fortement dépendante de leur environnement lequel, souvent incertain, exige flexibilité stratégique et réactivité des décisions (Raymond et St-Pierre, 2005).

L'objectif exploratoire de notre contribution est donc d'initier une réflexion autour de la question de recherche suivante: le BSC est-il applicable aux PME qui souhaitent mettre en place un système de management de leur performance stratégique? Cette question nous semble utile dans la mesure où le $\mathrm{BSC}$ pourrait répondre à de nouveaux besoins en émergence. Elle nous semble également intéressante parce qu'elle interroge la contingence d'un modèle présenté comme universel par ses auteurs.

\section{Les avantages et les limites du BSC en PME : un délicat compromis}

Cette question de l'application du BSC aux PME est très peu documentée. Quelques auteurs recommandent aux PME d'utiliser le BSC, pour accroître leur compétitivité et faire face à l'incertitude de leur environnement (Tennant et Tanoren, 2005 ; Fernandes et al., 2006; Gumbus et Lussier, 2006 ; Naro, 2006 ; Santin et Van Caillie, 2006). Ils soulignent les avantages suivants: le BSC motive le dirigeant à formaliser davantage sa stratégie ; il lui apprend à choisir un système de mesures multidimensionnel aligné sur cette dernière ; il focalise son attention sur un management dynamique des processus et des facteurs de performance.

Les quelques recherches empiriques qui analysent le taux de diffusion du BSC aux PME, et ce dans différents pays, constatent cependant que ce dernier est très faible (Germain, 2005; Tennant et Tanoren, 2005 ; Rautenstrauch, 2006 ; Sousa et al., 2006). Ce constat reflète probablement le fait que le BSC n'est que très peu connu des PME. Mais il pourrait aussi refléter le fait que le BSC ne correspond pas à leurs besoins. Plusieurs auteurs évoquent en effet des barrières récurrentes à sa mise en œuvre dans ce contexte : un manque de ressources financières, un manque de temps et de ressources humaines compétentes, des problèmes de disponibilité des données dans les systèmes d'information (Hudson et al., 2001 ; Garengo et al., 2005 ; Tennant et Tanoren, 2005). Ils soulignent également la difficulté qu'il y a à concilier une démarche perçue comme «complexe», "mécanisée », « déterministe », avec les besoins de « simplicité », de « flexibilité » et de « réactivité » spécifiques aux PME (McAdam, 2000 ; Germain, 2005 ; Naro, 2006). 
En résumé, les conclusions de la littérature sont contrastées mais laissent penser que le BSC pourrait s'appliquer aux $\mathrm{PME}$ à condition de trouver un compromis entre, d'une part le coût et les contraintes de sa mise en œuvre et, d'autre part, les avantages structurants qu'il procure. Cependant la littérature ne donne, à notre connaissance, aucune piste concrète pour gérer ce difficile compromis. Quelques rares auteurs avancent des propositions théoriques pour adapter le BSC aux PME, mais n'en donnent pas de validation empirique et se limitent le plus souvent à changer le nom des axes du modèle ou leur sens de représentation (Garengo et al., 2005 ; Naro, 2006).

Selon nous, la question gagnerait à être traitée de manière inductive à partir d'une analyse des pratiques des PME et de leurs acteurs. Aussi avons-nous initié un programme de recherche dont l'objectif était d'explorer les questions suivantes: quels sont les besoins des PME en matière de systèmes de management de la performance stratégique? Existe-t-il des systèmes de management de la performance stratégique dans les PME et si oui, comment sont-ils conçus et utilisés ? (Oriot et al., 2010). Nous rendons compte ici d'une étude de cas, issue de ce programme de recherche, qui permet d'explorer plus spécifiquement la question de l'applicabilité du BSC aux PME.

\section{II - MÉTHODE DE RECHERCHE ET PRÉSENTATION DU CAS}

\section{Méthode de recherche}

Notre programme de recherche se fonde sur la méthode qualitative des études de cas, afin de comprendre les systèmes de management de la performance dans leur contexte spécifique, à partir des interprétations qu'en donnent les acteurs (Stake, 2005). Nous avons étudié plusieurs PME françaises, sélectionnées avec l'aide de partenaires professionnels, parce qu'elles avaient exprimé le besoin d'un système de management de leur performance stratégique, et initié en ce sens une démarche de formalisation de leur système de contrôle. Ces PME avaient entre 50 et 250 salariés, un CA inférieur à 50 millions d'euros et un total de bilan inférieur à 43 millions d'euros, selon les critères de la Commission européenne (European Commission, 2003). Elles étaient toutes juridiquement indépendantes, afin de ne pas étudier des pratiques qui ne seraient que le prolongement de celles d'une maison mère. Nous avons choisi d'exposer ici le cas de l'une de ces PME, que nous avons nommée Micro S.A. à des fins de confidentialité.

\section{Brève présentation du cas Micro S.A.}

L'entreprise Micro S.A. a été créée en 2005 par l'actuel dirigeant, Monsieur Benoît ${ }^{2}$, qui a racheté les actifs d'une société familiale en faillite. À son arrivée, Monsieur Benoît trouve une entreprise «moribonde ${ }^{3}$ » qu'il est urgent de redresser : le parc de machines est ancien, les employés sont démotivés et les meilleurs risquent de partir. Toutefois, l'entreprise dispose d'un savoir-faire industriel reconnu et d'un potentiel commercial. Spécialisée dans le traitement de surface de pièces destinées aux constructeurs de l'industrie automobile et à leurs équipementiers, elle emploie 90 salariés. Géographi-

2. Le nom du dirigeant a été changé à des fins de confidentialité.

3. Les expressions entre guillemets et en italique correspondent à des citations extraites des entretiens. 


\section{SÉLECTION DU CAS ÉTUDIÉ ET COLLECTE DES DONNÉES}

\section{Un cas exploratoire singulier mais porteur d'enseignements}

La probabilité d'identifier en France une PME ayant mis en place un BSC est relativement faible (Germain, 2005). Le cas Micro $S A$ a été choisi parce que son dirigeant était le seul, parmi les chefs d'entreprise rencontrés, à connaître le BSC et à avoir participé à sa mise en œuvre dans une autre PME où il travaillait précédemment.

Or, ce dirigeant a volontairement choisi de ne pas mettre en place le BSC chez Micro S.A. En capitalisant sur son expérience antérieure, il a « inventé » un système de management de la performance, qui l'a aidé à redresser l'entreprise après plusieurs années de perte.

Le contexte de cette PME est spécifique, et le profil de son dirigeant l'est aussi. Néanmoins, l'expérience relativement « rare » (uncommon, Stake, 2005) de ce dirigeant offre l'opportunité d'un terrain d'étude pertinent pour explorer notre question de recherche. Aussi nous sommes-nous efforcées de capter la « singularité » (uniqueness, Stake, 2005) de ce cas, sans renoncer à ses enseignements potentiels pour d'autres cas.

\section{Recueil et analyse des données}

Afin de comprendre pourquoi le dirigeant de Micro S.A. n'a pas retenu le BSC et comment il a mis en œuvre son système de management de la performance stratégique, des entretiens semi-directifs ont été conduits in situ. C'est avec le dirigeant, également directeur commercial et DRH, que nous avons conduit les entretiens les plus approfondis (3 entretiens d'une durée moyenne de 3 heures), compte tenu d'une part, de sa place centrale dans la définition de la stratégie et dans la conception du système de management de la performance, d'autre part, de son expérience antérieure de mise en place d'un BSC en PME. Par souci de triangulation (Stake, 2005), nous avons également conduit 1 entretien avec chacun des 3 membres du comité de direction (directeur de production, DAF, responsable méthodes et processus). Tous les entretiens ont été enregistrés et retranscrits. Ils ont été complétés d'une analyse documentaire (tableau de bord, comptes rendus de réunions, outils de communication, etc.) et d'une visite du site de production.

Une analyse de contenu thématique a ensuite été conduite par chacun des chercheurs, qui ont comparé leurs interprétations, afin d'affiner leur compréhension du cas. Ces interprétations ont été confrontées à celles des interviewés afin de les valider.

quement, elle se situe sur la route entre les usines de ses clients et celles de leurs fournisseurs de pièce, ce qui est un atout concurrentiel pour le traitement de pièces volumineuses dont le transport coûte cher. Elle évolue sur un marché de niche et ses concurrents sont de moins en moins nombreux, compte tenu des investissements requis pour rester compétitif. À son arrivée, l'intention du dirigeant est d'abord de « relancer l'entreprise en expliquant au personnel les enjeux essentiels à sa survie » et de « retrouver la confiance perdue des clients grâce à la motivation et à la réactivité du personnel ». 
Il souhaite ensuite se diversifier auprès d'autres segments de clientèle pour « pérenniser l'avenir » et "être moins dépendant des constructeurs automobiles ». Âgé de 48 ans, titulaire d'un diplôme d'ingénieur, Monsieur Benoît a fait une grande partie de sa carrière dans des groupes industriels internationaux et a occupé des fonctions variées avec une très bonne connaissance des démarches qualité. Il a volontairement choisi de quitter le monde de la grande entreprise pour celui des PME lorsqu'on lui a demandé de fermer des usines qu'il venait de restructurer. Il a d'abord pris un poste de responsable $R \& D$ dans une autre PME de la région, où il a participé à la mise en place d'un BSC en tant que membre du comité de direction, puis il a quitté ce poste pour racheter Micro S.A.

\section{III - ENSEIGNEMENTS DU CAS : ESQUISSE D'UN TABLEAU DE BORD STRATÉGIQUE «SUR-MESURE » POUR LE DIRIGEANT DE PME}

L'analyse du cas exploré questionne, d'une part, l'applicabilité du modèle normatif du BSC en PME et esquisse, d'autre part, le portrait d'un tableau de bord stratégique «sur-mesure» modelé sur les besoins du dirigeant de la PME étudiée, fruit d'un processus créateur de sens pour ses collaborateurs.

\section{Un tableau de bord "sur-mesure » pour dépasser le formalisme du BSC}

À son arrivée chez Micro S.A., Monsieur Benoît trouve une entreprise «qui fonctionne à l'aveugle » en matière de contrôle de gestion. Du fait de son expérience en grand groupe, il ressent le besoin de professionnaliser le management de cette PME et met donc immédiatement en place ce qu'il appelle «mon tableau de bord ». Il est le
« porteur du projet » et ne veut surtout pas faire appel à une société de conseil. S'il connaît le BSC, il ne souhaite cependant pas s'y référer explicitement et critique l'usage excessivement formalisé qui en avait été fait dans son précédent poste en PME, sous l'influence d'un cabinet de conseil. Ce dernier avait, en effet, suivi toutes les étapes d'un modèle préformaté utilisé dans les grandes entreprises : «Je m'occupais de la RD et le consultant m'avait collé, à moi tout seul, 7 indicateurs. J'y passais la moitié de la semaine ! J'avais autre chose à faire. Ça marche pas comme ça en PME ». Longue et coûteuse, nonassociée à un réel besoin, cette mise en œuvre de BSC s'était soldée par «un échec » : bon nombre d'indicateurs « séduisants sur le papier $»$ n'avaient jamais pu être alimentés par le système d'information et le dirigeant avait perdu la confiance de son management qui redoutait « quelque chose de monstrueux, trop de contrôle !»

Chez Micro S.A., dans un contexte de survie où le temps est compté, les ressources limitées et la confiance du management particulièrement précieuse, Monsieur Benoît n'a donc pas souhaité appliquer cette démarche dont il redoute le trop grand formalisme. Il ne dispose en effet ni du système d'information, ni des compétences humaines nécessaires, pour faire vivre tous les indicateurs qu'exigerait le modèle normatif. $\mathrm{Ce}$ cas suggère ainsi que l'application du BSC aux PME peut se heurter à une limite de temps et de ressources. Il invite également à penser que la rigidité de la démarche est peu compatible avec les exigences de flexibilité stratégique et de réactivité de décision, qui caractérisent les PME en environnement incertain (Raymond et St-Pierre, 2005). Le dirigeant de PME a besoin de 
piloter son entreprise de manière dynamique, de faire évoluer sa stratégie. Or, le BSC exige un investissement lourd, qu'il est difficile de renouveler fréquemment et qui risque de générer une représentation moins évolutive (Oriot et Misiaszek, 2004). Il semblerait donc que le BSC soit difficile à appliquer, tel quel, en PME du fait de sa préformalisation pensée par et pour les grandes entreprises. Ainsi, Monsieur Benoît préfère concevoir lui-même un tableau de bord « sur-mesure ", évolutif en fonction de ses besoins d'information prioritaires. En PME, le management est centralisé et personnalisé par le dirigeant (Julien, 2005). Aussi n'est-il guère étonnant que ce tableau de bord soit enraciné dans la vision que Monsieur Benoît a de l'entreprise, modelé sur ses besoins, ses expériences antérieures et son style de management. Il considère son tableau de bord avant tout comme le reflet des enjeux vitaux auxquels son entreprise doit faire face pour se redresser. Il le considère également comme un outil de communication sélective vis-à-vis de managers éprouvés par la faillite, qu'il faut redynamiser, responsabiliser et faire évoluer vers une culture de la performance. Il souhaite incarner un nouveau style de management, davantage rationnel, en rupture avec celui du dirigeant précédent, purement intuitif et dépourvu de vision stratégique. Enfin, il est attentif à ne pas fragiliser la confiance de l'équipe dirigeante en rigidifiant trop le contrôle. Il évite soigneusement de parler du BSC et estime l'expression tableau de bord plus neutre et mieux à même de favoriser la communication avec ses collaborateurs.
En conclusion, au-delà du manque de ressources et de l'exigence de souplesse déjà mis en avant dans la littérature, ce cas contribue à souligner que le formalisme du BSC peut également se heurter au principe de confiance, si essentiel en PME (Julien, 2005). Certaines dérives dans la formalisation du contrôle peuvent en effet compromettre la confiance et la cohésion organisationnelle (Guibert et Dupuy, 1997). Norreklit (2000) avait déjà souligné que le BSC aurait du mal à être accepté des organisations dont il ne parlait pas le langage et ne reflétait pas les structures de pensée des managers. En effet, un modèle préformaté ne saurait être aussi "créateur de sens » (Weick, 2001) qu'un tableau de bord surmesure, fruit d'un processus de construction collective porté par les acteurs en interne et fondé sur leurs besoins réels.

\section{Une combinaison d'indicateurs et de mécanismes informels, reflet de la logique de performance stratégique du dirigeant}

Lorsqu'il construit son tableau de bord surmesure, Monsieur Benoît capitalise toutefois sur sa connaissance du BSC, qu'il estime indéniablement structurant, parce qu'il incite à formaliser les objectifs stratégiques et à leur associer un système cohérent d'indicateurs. Ainsi, il clarifie d'abord ses enjeux stratégiques, puis choisit ses indicateurs en conséquence. En ce sens, son tableau de bord peut être qualifié de stratégique $^{4}$. Cependant, il n'utilise ni le langage du BSC, ni ses catégorisations normatives. Il souhaite rationaliser son management sans le «bureaucratiser» et joue donc un rôle de «filtre critique» par rapport au

4. L'expression « tableau de bord stratégique » utilisée dans ce texte ne correspond pas à la traduction de « Balanced Scorecard »; nous parlons ici de « tableau de bord stratégique sur-mesure du dirigeant ». 
BSC, en flexibilisant en quelque sorte la démarche formalisée.

Tout d'abord, plutôt que de définir la « mission », les «valeurs » et la «vision» de l'entreprise, puis d'en déduire des « objectifs stratégiques » présentés selon les quatre perspectives du BSC (Kaplan et Norton, 2001), Monsieur Benoît formule directement un petit nombre d' " objectifs stratégiques prioritaires ». Mais il ne parle ni de « stratégie de croissance », ni de « stratégie de productivité » (Kaplan et Norton, 1996). Ces construits de stratégies génériques sont en effet rarement mobilisés par les dirigeants de PME, qui préfèrent parler de " priorités stratégiques » (Oriot et Bergeron, 2011). Ces priorités correspondent ici aux résultats attendus pour redresser l'entreprise et assurer sa pérennité. D'une part, développer l'activité en fidélisant les clients et en se diversifiant afin de réduire la dépendance vis-à-vis du secteur automobile ; d'autre part, rationaliser les coûts et sélectionner les clients rentables. De même, Monsieur Benoît n'a pas voulu formaliser par écrit son diagnostic stratégique de peur d'entraver la réactivité de décision de ses managers. Mais il consacre beaucoup de temps « à se mettre bien d'accord » avec eux «sur ces enjeux vitaux simples ». Enfin, de manière à focaliser leur attention sur ces priorités, il associe systématiquement, à chacun des objectifs, un indicateur sur son tableau de bord.

Pour atteindre ces objectifs, Monsieur Benoît identifie ensuite des processus clés et des leviers de performance. Mais il ne cartographie pas exhaustivement tous les processus, il sélectionne les deux plus importants : le processus de production et le processus commercial de prospection des clients hors secteur automobile. De même, il sélectionne quelques leviers critiques de performance : la réactivité aux demandes clients, la qualité des prestations, la confiance client, la motivation du personnel. Ces facteurs de performance ne sont pas classés dans les quatre perspectives du BSC, mais ils auraient pu l'être...

Enfin, il ne formalise pas de carte stratégique (Kaplan et Norton, 2001), même s'il établit clairement des relations entre ces leviers de performance et les objectifs stratégiques : "Pour s'en sortir, il faut déjà avoir le CA et ça, c'est une action commerciale. On a vendu la qualité de notre savoirfaire, notre réactivité, être capable de répondre à une commande même le WE. Il faut avoir la qualité et avoir la confiance du client, ce qui nous permet de vendre plus cher que les concurrents et d'être informés en premier lors de nouveaux marchés. Et pour obtenir la confiance des clients et la garder, il faut que les salariés aient envie de vous suivre, qu'ils soient motivés. » Monsieur Benoît explique comment il interprète la logique de performance stratégique qui sous-tend son organisation et qui s'exprime sous la forme de relations entre des fins et des moyens (Norreklit, 2000). Mais il n'utilise pas les relations de causalité génériques du BSC.

Sur son tableau de bord, Monsieur Benoît associe ensuite des indicateurs à certains de ces processus et leviers de performance, mais pas à tous systématiquement, comme le préconise le BSC. En effet, il préfère utiliser des mécanismes informels de contrôle et de coordination comme substituts ou comme compléments à certaines mesures. Ainsi, au processus de production, il fait correspondre des indicateurs de productivité main-d'œuvre, machine et matière, indicateurs prospectifs de la structure de 
coûts. Ces indicateurs ont l'avantage d'être facilement mesurables avec le système d'information existant.

Par contre, il n'associe pas d'indicateur au processus commercial de prospection. Il préfère en effet superviser personnellement les actions de prospection de ses commerciaux, en organisant périodiquement des réunions, au cours desquelles ils apprennent ensemble à comprendre les besoins des nouveaux clients hors secteur automobile, et identifient parfois des opportunités stratégiques. Monsieur Benoît parle de ce mode de contrôle comme « du seul indicateur », dont il souhaite disposer, alors même qu'il n'y a pas de mesure ! La supervision directe peut ici se substituer à l'indicateur, parce que les commerciaux sont peu nombreux et que le dirigeant connait bien la fonction commerciale.

Pour suivre la réactivité aux demandes clients et la qualité des prestations, Monsieur Benoît utilise de manière complémentaire des indicateurs et des mécanismes informels de coordination et de contrôle. Il mesure, d'une part, les « retards de livraison » et la « non-conformité produits », autrement dit le risque de détérioration potentielle de ces facteurs. Il mobilise, d'autre part, des mécanismes informels de coordination qui « font office d'indicateurs précurseurs » et qui lui permettent de détecter plus rapidement les problèmes potentiels. La réactivité est ainsi gérée en amont grâce à des relations étroites tissées avec les clients, et favorisées par la proximité géographique, ce qui accroît la prévisibilité des commandes. De même, le dirigeant anticipe l'évaluation de la qualité en organisant pour ses clients, des démonstrations fréquentes du savoir-faire industriel, des visites de l'usine, des rencontres avec le personnel expert.
Enfin, sur les deux variables subjectives de la confiance client et de la motivation du personnel, Monsieur Benoît a choisi de substituer aux indicateurs des mécanismes informels de coordination et de contrôle. Dans le BSC de son ancienne PME, la confiance client était mesurée par une enquête de satisfaction sur la fiabilité de laquelle il émet des doutes. Chez Micro S.A., plutôt que d'imaginer une mesure arbitraire, il préfère interroger personnellement ses clients, avec qui il a tissé un réseau relationnel pérenne. De même, tant qu'il n'a qu'un seul site à gérer, il préfère multiplier les échanges de face à face avec son personnel, expliquer à chacun ses responsabilités vis-à-vis du client, plutôt que de mesurer arbitrairement la motivation.

Dans la littérature, les auteurs qui recommandent le BSC aux PME soulignent le risque d'un déficit d'indicateurs sur les variables immatérielles, mais n'y voient que la conséquence d'un manque de ressources et le signe d'un contrôle pauvre (Garengo et al., 2005 ; Tennant et Tanoren, 2005). Au contraire, nous montrons que ce peut être un choix délibéré du dirigeant que de ne pas tout mesurer et de capitaliser sur ses avantages spécifiques, puisqu'en PME, la coordination de proximité entre acteurs peut souvent se substituer à la mesure. L'important est que chaque variable clé soit bien sous contrôle, quel que soit le mode de contrôle utilisé.

En conclusion, alors que le BSC exige d'associer systématiquement une mesure à chaque objectif et à chaque facteur de performance, postulant qu'on ne gère bien que ce que l'on mesure (Trébucq, 2011), c'est dans une combinaison complémentaire d'indicateurs et de mécanismes informels de coordination et de contrôle que peut se 
refléter la logique de performance stratégique du dirigeant de PME. Dans la PME étudiée, cette moindre formalisation est un choix, le paradoxe étant que le dirigeant réinvente en quelque sorte, sans le dire et sans la représenter, une carte stratégique flexibilisée, où contrôle formel et contrôle informel s'ajustent et se complètent.

\section{Un choix sélectif d'indicateurs focalisés sur les besoins prioritaires du dirigeant}

Le tableau de bord du dirigeant de Micro S.A. est composé d'indicateurs financiers et d'indicateurs non financiers, lesquels sont en nombre plus important. Ces derniers reflètent, d'une part, la polyvalence du dirigeant de PME (Julien, 2005), qui intervient aussi bien sur des activités opérationnelles que sur des décisions stratégiques. Ils reflètent, d'autre part, ses multiples fonctions (DG, directeur commercial et DRH), la PME se caractérisant par un faible degré de spécialisation des managers (Julien, 2005). Monsieur Benoît utilise ainsi certains de ses indicateurs à différentes finalités, ce qui lui permet aussi d'en limiter le nombre: par exemple, l'indicateur « CA/effectif » lui permet d'évaluer tout à la fois la disponibilité du personnel et les besoins en personnel intérimaire, de prévoir le niveau de CA qu'il doit dégager pour couvrir les coûts salariaux et enfin de mesurer la productivité de sa main-d'œuvre.

Le dirigeant de Micro S.A. n'a pas choisi ses indicateurs en fonction des quatre catégories prédéterminées du BSC et lorsqu'on lui demande s'il pourrait s'y référer a posteriori, il ne parvient pas à arbitrer entre les différents classements possibles pour certains de ses indicateurs. Ces catégories normatives ne font pas sens pour lui. En effet, le système décisionnel du dirigeant de PME est fortement intégré et transversal, peu compatible avec la logique de cloisonnement fonctionnel (Naro, 2006), qui correspond au découpage du BSC en quatre axes. Monsieur Benoît explicite, en revanche, d'autres logiques de catégorisation qui semblent mieux correspondre à ses besoins et intentions d'usage. Il mobilise d'une part une logique de seuil de rentabilité et d'autre part un découpage entre facteurs de performance externes et facteurs de performance internes (activité commerciale et clients d'un côté, ressources humaines et ressources de production de l'autre). Ces catégories sont proches de celles déjà identifiées en PME par Bergeron et St-Pierre (2005). Elles confirment que la satisfaction clients est une mission prioritaire du dirigeant de PME (McAdam, 2000; Garengo et al., 2005), alors que le BSC poursuit, à l'origine, une finalité actionnariale (Norreklit, 2000).

Enfin, le dirigeant sélectionne ses indicateurs, en se concentrant sur ce qui ne va pas bien ou qui fait peser un risque sur la performance ou sur les relations clients (pertes matières, pannes machines, non-conformité des produits, retards de livraison, etc.). En effet, même si certains dysfonctionnements sont connus, ils doivent être mesurés sur le tableau de bord du dirigeant pour être reconnus de tous. Dans ce cas, l'indicateur a plus pour finalité de communiquer le problème que de l'identifier et il est utilisé pour donner sens au plan d'action visant à le résoudre. Porteur d'un message, il « crée du sens » (Weick, 2001) en rendant repérable une certaine réalité. Par exemple, chez Micro S.A. certains indicateurs ont permis de mesurer objectivement des problèmes d'organisation de la production ou de gaspillage des matières premières détectés par 
supervision directe et de justifier les plans d'action visant à les résoudre.

À l'inverse, Monsieur Benoît refuse d'utiliser certains indicateurs classiques du BSC des grandes entreprises (Kaplan, 2009), comme le turnover ou l'absentéisme, parce qu'ils ne correspondent pas, chez lui, à un dysfonctionnement. Naro (2006) souligne lui aussi que ce type d'indicateur peut être contreproductif en PME : le management risque en effet d'y voir un excès de contrôle. En outre, contrairement aux grandes entreprises, le dirigeant de PME dispose souvent de l'information avant même d'avoir la mesure, son lien aux activités opérationnelles étant plus étroit. Son besoin n'est donc pas aussi systématique que le laisse penser le modèle normatif du BSC.

Enfin, Monsieur Benoît a le souci de faire évoluer ses indicateurs sans les accumuler. Veillant à ne pas sédimenter les mesures, il a sélectivement retenu 9 «indicateurs chocs », afin de focaliser l'attention des managers sur les priorités: "Pour une PME, l'information détaillée est un luxe, on n'a pas le temps. Dans le BSC de ma PME précédente, 35 indicateurs avaient été identifiés. Il y en avait tellement, je savais plus ce que ça indiquait. Ça n'a pas de sens. » Le BSC, lorsqu'il exige de mesurer toutes les dimensions de la performance en n'utilisant que peu d'indicateurs, enferme en effet les managers dans une injonction contradictoire : être exhaustif tout en étant sélectif. En combinant indicateurs et mécanismes informels de contrôle, le dirigeant de PME peut inventer une réponse à cette double exigence d'exhaustivité et de sélectivité, parce que contrôler ne se réduit pas à mesurer. Les indicateurs sont utilisés pour souli- gner les priorités stratégiques et les problèmes urgents à résoudre. Tout mesurer reviendrait à dire que tout est important, de la même manière, au même moment, et donc que rien n'est vraiment important au bout du compte.

\section{Un usage interactif du tableau de bord pour un apprentissage créateur de sens}

Le modèle des leviers de contrôle de Simons (1995) différencie les systèmes de contrôle diagnostic, qui permettent le déploiement d'une stratégie préexistante et stable, des systèmes de contrôle interactif, qui favorisent la formation de stratégies émergentes via des processus d'apprentissage. Conçu à l'origine comme un système de contrôle diagnostic (Kaplan et Norton, 1992 ; Simons, 1995), le BSC a ensuite été également présenté comme un levier de contrôle interactif par ses auteurs (Kaplan, 2009). Mais les avis divergent et le débat est loin d'être clos. Comme le résument Naro et Travaillé (2011), si le BSC semble pouvoir être mobilisé autant comme un système de contrôle diagnostic que comme un système de contrôle interactif, c'est parce qu'il porte les représentations contingentes de ses concepteurs, comme de tous ceux qui l'utilisent et qui, ce faisant, se l'approprient. Par conséquent, il convient de comprendre, pour chaque cas, l'utilisation qui en est faite et les objectifs qui lui sont assignés.

Ainsi, dans la PME où Monsieur Benoît était précédemment, le BSC avait une finalité de contrôle diagnostic: le cabinet de conseil avait recommandé d'associer des valeurs cibles de performance à chaque indicateur, d'indexer la rémunération des managers sur l'atteinte de ces valeurs, puis de corriger les éventuelles déviations. Chez 
Micro S.A., Monsieur Benoît ne suit pas ces recommandations pour construire son tableau de bord. En effet, elles ne correspondent pas d'une part, à son besoin de flexibilité stratégique et, d'autre part, à son souci de préserver la confiance de ses collaborateurs. Avec des valeurs cibles systématiques, qui seraient perçues comme un excès de contrôle, il redoute de reproduire la rigidité du BSC de son ancienne PME. Il choisit donc de n'attribuer de valeurs cibles qu'à certains de ses indicateurs, de manière sélective, afin de souligner leur importance et afin de garder la souplesse nécessaire pour les ajuster facilement. Pour les mêmes raisons, il n'indexe pas les rémunérations sur l'évolution des indicateurs, craignant d'inciter les managers à manipuler les mesures : «Le grand danger d'un indicateur c'est que si les gens le vivent comme un outil de pression, ils vont essayer de se débrouiller pour avoir de bons résultats. Et vous avez tout faux ». En outre, strictement utilisé comme un levier de contrôle diagnostic, le BSC risque de cloisonner la conception de la stratégie de sa mise en œuvre, le temps de la réflexion de celui de l'action (Naro, 2006), ce qui n'a guère de sens en PME, où stratégies délibérées et stratégies émergentes doivent pouvoir se conjuguer en permanence (Mintzberg et al., 1999 ; Naro et Travaillé, 2010).

Ainsi, en reprenant Micro S.A., dans un contexte relativement instable, le besoin qu'exprime Monsieur Benoît est davantage celui d'un système de contrôle interactif (Simons, 1995), qui focalise l'attention de ses collaborateurs sur les incertitudes stratégiques, de manière à faire émerger des opportunités, avec leur aide et avec leur adhésion. Il construit donc un tableau de bord sur-mesure par rapport à cette finalité d'usage. À son arrivée, dans l'urgence, certes, il définit d'abord seul, ses priorités stratégiques et son tableau de bord, mais à aucun moment il n'impose autoritairement l'outil. Ensuite, grâce aux discussions qu'il anime autour des indicateurs, les managers s'approprient le tableau de bord, qui devient progressivement une référence pour toute l'équipe dirigeante. Ces discussions collégiales sont aussi l'occasion de revisiter la stratégie initiale et de donner sens à l'objectif de diversification : certains marchés sont écartés car trop risqués, d'autres opportunités de marchés émergent. De même, les indicateurs révèlent des clients non rentables, et Monsieur Benoît renégocie les prix, ce que ses collaborateurs pensaient impossible jusqu'alors. L'utilité du tableau de bord est ainsi confirmée par le directeur de production : «On a beaucoup discuté et on a fait le ménage. On a sorti les produits qui ne rapportaient rien, on a augmenté les prix de vente, et Monsieur Benoît a su tenir tête aux constructeurs, parce qu'on avait enfin la preuve qu'ils menaçaient notre rentabilité. On a décidé ensemble d'éliminer les clients non rentables et de développer d'autres marchés. » Les indicateurs questionnent en effet les représentations que les managers avaient des relations aux clients, jusqu'alors figées dans des habitudes passives, et légitiment leur évolution. Ainsi, les acteurs ressourcent leur système interprétatif et le remettent en cause de manière continue (Weick, 2001). La stratégie devient un processus de construction collective, porteur de sens. De même, les débats autour des indicateurs favorisent l'apprentissage par Monsieur Benoît des métiers de sa nouvelle entreprise, des savoirs opérationnels que ses collaborateurs maîtrisent et qu'ils lui font partager. 
En conclusion, ce cas invite à penser qu'un usage interactif des tableaux de bord stratégiques en PME (Naro, 2006 ; Messeghem et al., 2010) pourrait être privilégié dans un contexte de forte incertitude, quand il est essentiel de préserver la cohésion de l'équipe dirigeante et son aptitude à changer rapidement d'orientation stratégique. Flexibilité stratégique et réactivité sont alors favorisées par une moindre formalisation, par des circuits de décisions courts et transverses, par la polyvalence des managers et par leur forte implication dans les activités opérationnelles. Ce cas nous suggère aussi que les systèmes de pilotage stratégiques des PME, quels qu'ils soient, devraient être conçus sur-mesure par rapport aux intentions d'usage des dirigeants, lesquelles peuvent correspondre à différentes combinaisons de contrôle diagnostic et de contrôle interactif, susceptibles d'évoluer dans le temps. Ces systèmes de pilotage, enfin, ne valent que ce que vaut l'animation de gestion à laquelle ils servent de support et la dynamique d'apprentissage collectif qu'ils alimentent : ils ne sont que prétextes à être intelligents ensemble (Lorino, 1997).

\section{CONCLUSION}

Dans le cas exploratoire étudié, le BSC n'est donc volontairement pas appliqué. Le dirigeant, alors qu'il connaît le modèle et ses principes structurants, a choisi de ne pas s'y référer explicitement et joue un rôle de filtre critique en construisant un tableau de bord stratégique sur-mesure, outil qui l'aide à redresser l'entreprise et à redynamiser ses collaborateurs.

Nos résultats questionnent donc l'universalité du modèle normatif et incitent à penser que le BSC, tel que préformaté à l'origine pour les grandes entreprises et présenté comme standard par ses auteurs, n'est pas systématiquement applicable en PME. En effet, d'une part, sa mise en œuvre peut se heurter aux caractéristiques spécifiques des PME : ressources limitées, exigence de réactivité et de flexibilité stratégique. D'autre part, son excessif formalisme risque de mettre en danger la confiance du management, si cruciale en PME. Ce double constat est encore renforcé dans le cas étudié, celui d'une PME en redressement, où le nouveau dirigeant, en situation de forte incertitude, doit impérativement susciter l'adhésion de ses collaborateurs. Ce cas unique, bien que riche d'enseignements, ne nous permet cependant pas de généraliser nos résultats qui devront ultérieurement être mis à l'épreuve d'autres PME.

Nos résultats interrogent, toutefois, la transférabilité aux PME d'outils de gestion normés par et pour les grandes entreprises, renforçant un scepticisme déjà exprimé (Marchesnay, 1993 ; St-Pierre et Delisle, 2006), et ce d'autant plus que les PME n'ont pas toutes le projet de devenir grandes et d'adopter les outils des grandes. C'est aux systèmes de management de la performance de s'adapter aux réalités des différentes PME et non à ces dernières de passer sous les fourches caudines de modèles prédéfinis, quels qu'ils soient. En fait, compte tenu de leur contrainte de ressources et de leur exigence de flexibilité, les dirigeants de PME privilégient les outils dont ils ont véritablement besoin (St-Pierre et Delisle, 2006).

En ce sens, le cas étudié nous enseigne qu'un système de management de la performance stratégique, pour être utile au dirigeant de PME, doit être aligné sur ses besoins. Nous invitons donc les dirigeants 
de PME à construire des tableaux de bord stratégiques taillés sur-mesure par rapport à leur expérience, leur contexte spécifique et l'évolution de leurs priorités stratégiques, qui utilisent le langage de leurs collaborateurs et qui s'enracinent dans leurs représentations cognitives. Ces tableaux de bord gagneront à être le fruit d'un processus de construction collective porté par toute une équipe dirigeante.

Nos résultats invitent enfin à repenser les modes de diffusion des outils de gestion en PME et le rôle des prescripteurs, qui devraient s'attacher à bien comprendre et clarifier les intentions d'usage de chaque équipe dirigeante, sachant que toutes les PME n'ont peut-être pas besoin de systèmes de management de la performance stratégique. Celles qui en ont besoin se reconnaîtront peut-être dans le cas étudié, celui d'une entreprise confrontée à des changements importants et rapides, avec un nouveau dirigeant porteur de projets ambitieux, et qui a déjà une expérience des outils de gestion, y compris en grande entreprise. Le portrait que nous avons esquissé d'un tableau de bord construit surmesure pourra peut-être, en ce sens, aider les dirigeants de ces PME d'un type particulier à identifier, s'ils disposent ou non d'un système de management de la performance stratégique et sinon, leur donner des pistes pour en construire un. Nous leur suggérons alors de jouer sur une combinaison complémentaire d'indicateurs et de mécanismes informels de contrôle et de coordination, pour penser un système à la fois exhaustif et sélectif. Ce faisant, ils utiliseront leurs avantages spécifiques pour professionnaliser leur management, avec flexibilité, tout en initiant un processus créateur de sens pour leur organisation.

\section{Bibliographie}

Bergeron H., St-Pierre J. (2005). «Les indicateurs de performance financière et non financière: complémentarité ou substitution ? Étude exploratoire sur des PME manufacturières », Actes du $26^{e}$ Congrès de l'Association Francophone de Comptabilité, Lille.

Bourguignon A., Malleret V., Norreklit H. (2002). «L'irréductible dimension culturelle des instruments de gestion : l'exemple du tableau de bord et du balanced scorecard », Comptabilité Contrôle Audit, $\mathrm{n}^{\circ}$ spécial, mai, p. 7-32.

Chenhall R. (2005). "Integrative strategic performance measurement systems, strategic alignment of manufacturing, learning and strategic outcomes: an exploratory study", Accounting, Organizations and Society, 30, p. 395-422.

Choffel D., Meyssonnier F. (2005). «Dix ans de débats autour du Balanced Scorecard », Comptabilité Contrôle Audit, tome 11, vol. 2, décembre, p. 61-81.

European Commission (2003). "SME definition: Commission Recommendation 2003/361/EC of 06 May 2003”, Official Journal of the European Union L 124, p. 36.

Fernandes K., Raja V., Whalley A. (2006). "Lessons from implementing the balanced scorecard in a small and medium size manufacturing organization”, Technovation, vol. 26, p. 623-634. 
Garengo P., Biazzo S., Bititci U. (2005). "Performance measurement systems in SMEs: a review for a research agenda", International Journal of Management Review, vol. 7, $\mathrm{n}^{\circ} 1$, p. $25-47$.

Germain C. (2005). «Une typologie des tableaux de bord implantés dans les PME », Finance Contrôle Stratégie, vol. 8, $\mathrm{n}^{\circ}$ 3, septembre, p.125-143.

Guibert N., Dupuy Y. (1997). " La complémentarité entre contrôle "formel” et contrôle “informel” : le cas de la relation client-fournisseur », Comptabilité Contrôle Audit, tome 3, vol. 1, mars, p. 39-52.

Gumbus A., Lussier R. (2006). "Entrepreneurs use a Balanced Scorecard to translate strategy into performance measures", Journal of Small Business Management, vol. 44, n 3, p. $407-$ 425.

Hudson M., Smart A., Bourne M. (2001). "Theory and practice in SME performance measurement systems", International Journal of Operations and Production Management, vol. 21, n 8, p. 1096-1115.

Julien P.A. (2005). Sous la direction de, GREPME, Les PME : Bilan et perspective, $3^{\mathrm{e}}$ édition, Presses Inter Universitaires, Cap Rouge Québec.

Kaplan R., Norton D. (1992). "The Balanced Scorecard-measures that drive performance”, Harvard Business Review, vol. 70, $\mathrm{n}^{\circ}$ 1, January-February, p. 71-79.

Kaplan R., Norton D. (1996). The Balanced Scorecard: translating strategy into action, Harvard Business School Press, Boston.

Kaplan R., Norton D. (2001). The Strategy-Focused Organization: How Balanced Scorecard Companies Thrive in the New Business Environment, Harvard Business School Press, Boston.

Kaplan R. (2009). "Conceptual Foundations of the Balanced Scorecard", Handbook of Management Accounting Research, Chapman C., Hopwood A., Shields M. (Eds.), vol. 3, Elsevier, Oxford, p.1253-1269.

Lorino P., (1997). Méthodes et pratiques de la performance, Éditions d'Organisation, Paris.

Marchand M. (octobre 2009). L'utilisation des systèmes de mesures de performance dans les PME, Thèse de 1'Université du Québec Trois-Rivières.

Marchesnay M. (1993). « PME, stratégie et recherche », Revue française de gestion, $\mathrm{n}^{\circ} 95$, septembre-octobre, p.70-76.

McAdam R. (2000). "Quality models in an SME context", International Journal of Quality and Reliability Management, vol. 17, $\mathrm{n}^{\circ}$ 3, p. 305-323.

Méric J. (2003). « L'émergence d'un discours de l'innovation managérial. Le cas du Balanced Scorecard », Comptabilité Contrôle Audit, n spécial, mai, p. 129-145.

Messeghem K., Naro G., Samut S. (2010). «Construction d'un outil stratégique d'évaluation de l'accompagnement à la création d'entreprise : apport du tableau de bord prospectif », Gestion 2000, mars-avril, p. 95-112.

Mintzberg H., Ahlstrand B., Lampel J. (1999). Safari en pays stratégie. L'exploration des grands courants de la pensée stratégique, Village Mondial, Paris. 
Naro G. (2006). «Un tableau de bord stratégique pour les dirigeants de petites entreprises : apports et limites des tableaux de bord prospectifs », Fourcade C., Paché G., Pérez R. (éds), La stratégie dans tous ses états. Mélanges en l’honneur du Professeur Michel Marchesnay, EMS, Paris, p. 205-229.

Naro G., Travaillé D. (2010). « Construire les stratégies avec le Balanced Scorecard : vers une approche interactive du modèle de Kaplan et Norton », Finance Contrôle et Stratégie, vol. $13, \mathrm{n}^{\circ} 2$, juin, p. 33-66.

Naro G., Travaillé D. (2011). « Le BSC à l'épreuve de l'expérience. De l'assemblage d'un "puzzle" et construction d'un "Lego" », Revue française de gestion, vol. 37, n 211, p. $65-80$.

Norreklit H. (2000). "The Balance on the Balanced Scorecard - a critical analysis of some of its assumptions", Management Accounting Research, $\mathrm{n}^{\circ}$ 11, p. 65-88.

Oriot F., Bergeron H. (2011). « Indicateurs de performance et priorités stratégiques des dirigeants de PME », Le Grand Livre de l'Économie PME, Lecointre G. (sous la direction scientifique de), Gualino, Paris.

Oriot F., Misiaszek E. (2004). "Technical and organizational barriers hindering the implementation of a Balanced Scorecard : the case of a European Space Company", Performance Measurement and Management Control: Superior Organizational Performance, in the Series Studies in Managerial and Financial Accounting, Epstein M. \& Manzoni JF., vol. 14, Elsevier Science, Oxford, p. 265-301.

Oriot F., Otley D., Misiaszek E. (2010). "Strategic Performance Management Systems in SMEs: exploring new research opportunities", $31^{e}$ Congrès de l'Association Francophone de Comptabilité, Nice.

Rautenstrauch T. (2006). "Balanced Scorecard adoption in SME's: empirical evidence from a German perspective", 29th Annual Congress of the European Accounting Association, Dublin, 22-24 March.

Raymond L., St-Pierre J. (2005). “Antecedents and performance outcomes of advanced manufacturing systems sophistication in SMEs", International Journal of Operations \& Production Management, vol. 25, n 6, p. 514-533.

Santin S., Van Caillie D. (2006). « Diagnostic préalable à la transmission et stratégie de reprise de la PME : une relation de contingence forte », $8^{e}$ Congrès International Francophone en Entrepreneuriat et PME (CIFEPME), HEG Fribourg Suisse.

Simons R., (1995). Levers of Control. How managers use innovative control system to drive strategic reneval, Harvard Business School Press, Boston.

Sousa S., Aspinwall E., Rodrigues A. (2006). "Performance measures in English small and medium enterprises: survey results”, Benchmarking: An International Journal, vol. 13, $\mathrm{n}^{\circ} 1-2$, p. 120-134.

Stake R.E. (2005). "Qualitative Case Studies", Handbook of Qualitative Research, Denzin N.K. \& Lincoln Y.S. (Eds.), Sage Publication, London, p. 443-466.

St-Pierre J., Delisle S., (2006). “An expert diagnosis system for the benchmarking of SMEs' performance”, Benchmarking: An International Journal, vol. 13, n 1-2, p. 106-119. 
Tennant C., Tanoren M. (2005). "Performance management in SMEs: a Balanced Scorecard perspective", International Journal of Business Performance Management, vol. 7, $\mathrm{n}^{\circ} 2$, p. 123-143.

Torres O. (2007). «Approche descriptive de la spécificité de gestion des PME : le mix de proximité », Management des PME. De la création à la croissance, Filion L.J. (sous la direction de), Pearson, Québec.

Trébucq S. (2011). « Le Balanced Scorecard en France. Un outil de communication encore incompris », Revue française de gestion, vol. 37, n² 211, p. 131-143.

Weick K. (2001). Making Sense of the Organization, Blackwell, Massachusetts. 
